\title{
The Combination of CD147 and MMP-9 Serum Levels Is Identified as Novel Chemotherapy Response Markers of Advanced Non-Small-Cell Lung Cancer
}

\author{
Xiaojuan Qiao, ${ }^{1}$ Yan Gu, ${ }^{2}$ Jingfeng Yu, ${ }^{3}$ Jinghui Wang, ${ }^{4}$ Xuan Liu, ${ }^{5}$ Meng Gu, ${ }^{6,7}$ Li Ma, ${ }^{6,7}$ \\ Yongfeng Jia $\mathbb{D}^{8},{ }^{8}$ and Shucai Zhang $\mathbb{D}^{4}$ \\ ${ }^{1}$ Department of Medical Oncology, The Affiliated Hospital of Inner Mongolia Medical University, Hohhot, Inner Mongolia, China \\ ${ }^{2}$ Department of Respiratory Medicine, The Affiliated Hospital of Inner Mongolia Medical University, Hohhot, Inner Mongolia, China \\ ${ }^{3}$ College of Basic Medicine, Inner Mongolia Medical University, Hohhot, Inner Mongolia, China \\ ${ }^{4}$ Department of Medical Oncology, Beijing Chest Hospital, Capital Medical University, Beijing, China \\ ${ }^{5}$ Department of Surgery, The Affiliated Hospital of Inner Mongolia Medical University, Hohhot, Inner Mongolia, China \\ ${ }^{6}$ Department of Cellular and Molecular Biology, Beijing Chest Hospital, Capital Medical University, Beijing, China \\ ${ }^{7}$ Department of Cellular and Molecular Biology, Beijing Tuberculosis and Thoracic Tumor Research Institute, Beijing, China \\ ${ }^{8}$ Department of Pathology, The Affiliated Hospital of Inner Mongolia Medical University, Hohhot, Inner Mongolia, China
}

Correspondence should be addressed to Yongfeng Jia; jiayongfengfy@163.com and Shucai Zhang; zhangshucaibj@163.com

Received 17 January 2020; Revised 17 January 2020; Accepted 27 March 2020; Published 24 April 2020

Academic Editor: Hubertus Himmerich

Copyright (c) 2020 Xiaojuan Qiao et al. This is an open access article distributed under the Creative Commons Attribution License, which permits unrestricted use, distribution, and reproduction in any medium, provided the original work is properly cited.

To evaluate the correlation between the changes in serum concentrations of cluster of differentiation-147 (scCD147) and chemotherapy outcome in patients with NSCLC and evaluate the combination of scCD147 with serum matrix metalloproteinase-9 (scMMP-9) levels in the prediction of chemotherapy response, eighty-two patients with advanced LC were enrolled. Newly diagnosed cases were treated with platinum-based chemotherapy. We measured scCD147 protein levels in LC cases by ELISA and used receiver operating characteristic (ROC) curves to analyze the results. Four time points were chosen to examine the association between the changes in scCD147 and chemotherapy outcome: before chemotherapy and 21 days after the start of the first, second, and fourth chemotherapy cycles. We assessed the combination of scCD147 and scMMP-9 serum levels in predicting the chemotherapy response. scCD147 was higher in LC cases than that in healthy volunteers (HVs). scCD147 was associated with distant metastases and TNM stage. scCD147 and scMMP-9 appeared to be independent predictive factors for chemotherapy outcomes after the first and second chemotherapy cycles for patients with NSCLC. Multivariable analysis also demonstrated that variations in scCD147 and scMMP-9 could be independent factors for monitoring chemotherapy outcome for patients with NSCLC. Furthermore, when scCD147 and scMMP-9 are combined into a new risk model, it has a markedly better prediction of chemotherapy outcomes than each protein alone. scCD147 and MMP-9 are potential predictive biomarkers for efficacy, and their combination significantly improves the predictive power for chemotherapy response in patients with NSCLC.

\section{Introduction}

Lung cancer (LC) is the fifth leading cause of death in China [1]. LC can be of two main types: non-small-cell lung cancer (NSCLC) or small-cell lung cancer (SCLC) [2]. Overall survival at 5 years for LC has improved only slightly over the past 40 years [3]. The treatment is still challenging because of the invasion/metastases of tumor cells [4]. Although surgical resection sometimes cures early stage NSCLC, few therapeutic options are available for advanced-stage NSCLC, highlighting the importance of a better understanding of the disease to find novel therapeutic targets. Therefore, 
the identification of novel, predictive, and efficacious predictive biomarkers can help to deliver "individualized and efficient" therapy.

Several biomarkers have been found to be involved in the invasion/metastases of tumor cells [5]. Cluster of differentiation (CD) 147 (also known as "extracellular matrix metalloproteinase inducer" or "basigin") is a member of the immunoglobulin superfamily and was first observed on the surfaces of tumor cells [6,7]. CD147 is closely associated with the invasion/metastases of tumor cells [8]. With regard to the heterogeneity of tumor microenvironments, CD147 can activate matrix metalloproteinases (MMPs), degrade the extracellular matrix (ECM) of tumor cells, and induce the invasion/metastases of tumor cells $[9,10]$. CD147 also plays a pivotal role in inhibiting the apoptosis of tumor cells [11].

MMP-9 has been reported to play a crucial part in the invasion/metastases of tumor cells. MMP-9 can degrade type-IV collagen (which constitutes the ECM and basal membrane). Scholars have postulated that CD147 and MMP-9 could be unique biomarkers for type-II/III astrocyte-elevated genes and predict tumor progression and prognosis. However, the possibility of utilizing serum CD147 and MMP-9 levels as biomarkers has not been validated.

Previously, we showed that serum concentrations of MMP-9 in NSCLC cases during chemotherapy were intimately associated with chemotherapy outcome [12]. Analysis was performed based on the same dataset with the same methods as the previous publication. We evaluated the correlation between the changes in serum concentrations of CD147 and chemotherapy outcome in NSCLC cases and analyzed the relationship with the changes in serum concentrations of MMP-9. In this way, we assessed the possible role of the combination of serum CD147 and MMP-9 levels as biomarkers for evaluating chemotherapy outcomes.

\section{Materials and Methods}

2.1. Patients. Eighty-two cases (59 (72\%) males and 23 (28\%) females; median age, 60 (range, 20-79) years) with advanced LC admitted to Beijing Chest Hospital (Capital Medical University, Beijing, China) from August 2013 to October 2014 were enrolled. The diagnosis was confirmed by histology or cytology. The patients did not receive treatment before study commencement.

Cases were categorized as "smokers" $(65$ (79.3\%)) or "nonsmokers" (17 (20.7\%)). Additionally, 72 (87.8\%) cases had an Eastern Cooperative Oncology Group Performance Status (ECOG PS) score of $0-1$, whereas 10 (12.2\%) cases had an ECOG PS score of $\geq 2$. The number of patients with adenocarcinoma, squamous cell carcinoma (SCC), or SCLC was 35 (42.7\%), 18 (21.9\%), and 29 (35.4\%), and those with type-IIIa, type-IIIb, and type-IV disease totaled 8 (9.8\%), 17 $(20.7 \%)$, and $57(69.5 \%)$, respectively.

In $25(30.5 \%)$ cases, metastases had not occurred, whereas in 57 (69.5\%) individuals, distant metastasis had occurred. The number of patients with N0, N1, N2, and N3 stages was $8(9.8 \%), 3(3.7 \%), 23(28 \%)$, and $48(58.5 \%)$, whereas the number with T1, T2, T3, and T4 stages was 3
(3.7\%), 28 (34.1\%), 13 (15.9\%), and 38 (46.3\%), respectively. Analysis was performed based on the same dataset with the same methods as our previous publication [12]. This study was approved by the Ethics Committee, and all of the patients provided written informed consent.

Among 30 healthy volunteers (HVs), 18 (60.0\%) were male and $12(40.0 \%)$ were female. The median age was 57 (range, 26-68) years. Compared with 82 cases of LC, sex $(P=0.227)$ and age $(P=0.951)$ were not significantly different.

2.2. Chemotherapy and Assessment of Response. All cases received platinum-based treatment: cisplatin $\left(75 \mathrm{mg} / \mathrm{m}^{2}\right)$ on day 1 and day 2 , carboplatin area under curve 5 $\left(300-350 \mathrm{mg} / \mathrm{m}^{2}\right)$ on day 2 , and nedaplatin $\left(75 \mathrm{mg} / \mathrm{m}^{2}\right)$ on day 1. Different histologic types were treated with platinum and other drugs: paclitaxel liposomes $\left(150-175 \mathrm{mg} / \mathrm{m}^{2}\right.$ on day 1 ) or pemetrexed $\left(500 \mathrm{mg} / \mathrm{m}^{2}\right.$ on day 1 ) for adenocarcinoma and paclitaxel liposomes for SCC. The cycle was repeated every 21 days. We excluded patients if they had progressive disease (PD), if unbearable toxicity during chemotherapy was recorded, or if patients refused treatment.

Tumor diameter was measured using computed tomography before chemotherapy as well as 21 days after the start of the first, second, and fourth cycles of chemotherapy. The responses of patients were analyzed by the Response Evaluation Criteria in Solid Tumors based on a complete response (CR), partial response (PR), stable disease (SD), and PD.

The efficacy evaluation was divided into disease control (CR/PR/SD) and progressive disease (PD). In NSCLC patients, there were 47 cases with $C R / P R / S D$ and 6 cases with PD after the first cycle of chemotherapy. After the second cycle of chemotherapy, there were 37 cases with CR/PR/SD, 13 cases with PD ( 7 cases of PD were newly added in the second cycle), and 3 cases of shedding patients. After the fourth cycle of chemotherapy, there were 14 cases with CR/PR/SD, 25 cases with PD (12 cases of PD were newly added in the cycle), and 11 cases of shedding patients.

2.3. Measurement of Serum Concentrations of CD147. Blood samples were obtained before treatment for all patients. For patients with NSCLC, samples were collected at the four time points mentioned above. Serial samples were collected from 53 cases at the first and second cycles, from 44 at the third cycle, and from 26 at the fourth cycle. Each blood sample was centrifuged at $1509 \mathrm{~g} / \mathrm{min}$ for $5 \mathrm{~min}$ at room temperature, separated into plasma and supernatant, and stored at $-80^{\circ} \mathrm{C}$. Serum concentrations of CD147 were detected using an enzyme-linked immunosorbent assay (ELISA) kit based on the manufacturer's (USCN Life Science, Houston, TX, USA) protocols.

2.4. Statistical Analyses. Statistical analyses were performed using SPSS v19.0 (IBM, Armonk, NY, USA). The chi-squared $\left(\chi^{2}\right)$ test was carried out to ascertain the relationship between classification variables. Nonparametric statistical analyses were employed due to the broad data range. Serum levels of CD147 are given as median values and 


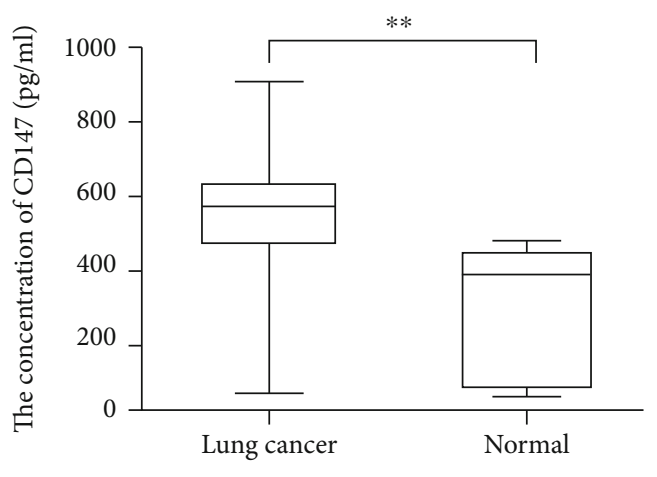

(a)

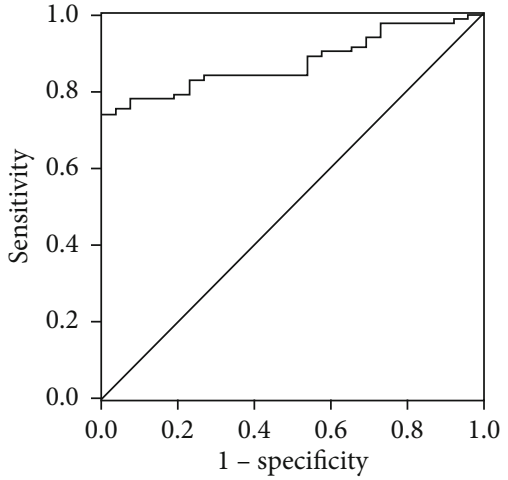

(b)

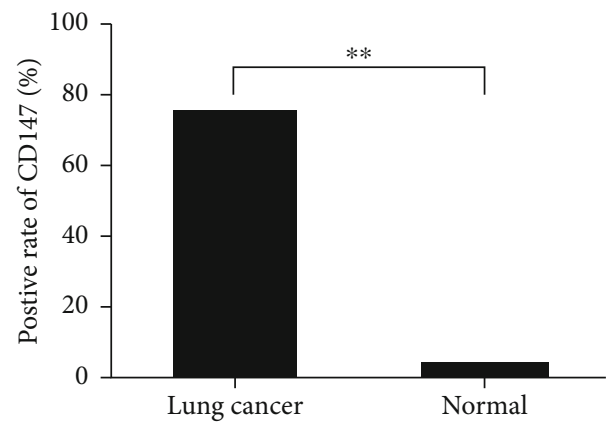

(c)

FIGURE 1: (a) Differences in the serum concentrations of CD147 between patients with lung cancer (LC) and healthy volunteers (HVs). (b) The diagnostic value of serum concentrations of CD147 for identifying LC in a receiver operating characteristic curve. (c) Differences in the positive rate of serum concentrations of CD147 between patients with LC and HVs. ${ }^{* *} P<0.01$.

ranges. The Mann-Whitney $U$-test was employed to analyze the difference between measurement parameters. A receiver operating characteristic (ROC) curve was created by SPSS v19.0. All tests were two-sided, and statistical significance was set at $P<0.05$.

For sensitivity and specificity analysis, the following score model was constructed: sensitivity $\%=($ true-positive rate $) /$ (true-positive rate + false-negative rate) $* 100 \%$; specificity $\%=$ (true-negative rate) $/($ true-negative rate + false-positive rate) $* 100 \%$.

The multivariate Cox proportional hazards regression model was used to evaluate independent factors associated with tumor response. As a multivariate analysis, all clinical factors were included in the model.

\section{Results}

3.1. Serum Concentrations of CD147 in LC Patients and HVs. Serum concentrations of CD147 in $82 \mathrm{LC}$ cases and $30 \mathrm{HVs}$ were measured by ELISA. The mean serum level of CD147 in LC cases was 563.77 (range, 50.32-910.80) pg/mL, which was much higher than that in HVs (381.05 (range: 43.38$474.52) \mathrm{pg} / \mathrm{mL})(P<0.001)$ (Figure 1(a)). ROC curves were employed to ascertain the serum concentrations of CD147. The area under the ROC curve (AUC) was 0.876 (95\% confidence interval (CI): 0.812-0.939). The difference in the AUC was significant compared with $0.5(P<0.001)$. When the cut-off value for the serum concentration of CD147 was $466.14 \mathrm{pg} / \mathrm{mL}$, the sensitivity was $75.6 \%$, the specificity was $96.2 \%$, the positive predictive value was $98.4 \%$, and the negative predictive value was $59.1 \%$ for the diagnosis of lung cancer (Figure 1(b)). Using a serum concentration of $466.14 \mathrm{pg} / \mathrm{mL}$ as the cut-off point, $62(75.6 \%)$ LC cases had a higher serum level of CD147, whereas only $1(3.3 \%)$ HV did, and this difference was significant $(P<0.001)$ (Figure 1(c)).

3.2. Correlation between the Serum Concentrations of CD147 and MMP-9 before Chemotherapy and Clinical Characteristics in NSCLC. The serum concentrations of CD147 in patients with LC were related to distant metastases and tumor-node-metastasis (TNM) stage. We did not find a significant association between the serum concentrations of CD147 and sex, age, smoking status, ECOG PS score, histology, diameter of primary tumor, or metastases to regional lymph nodes $(P>0.05)$. Serum concentrations of CD147 were significantly higher in cases with the M1 stage than concentrations in cases with the M0 stage $(P=0.037)$. Serum concentrations of CD147 in cases with stage-IV disease were significantly higher than those in cases with stage-III disease $(P=0.037)$.

There was no significant association between the serum concentrations of CD147 in patients with NSCLC and the clinical characteristics $(P>0.05)$ (Table 1$)$. The serum 
TABLE 1: The correlation between serum CD147 and MMP-9 levels before chemotherapy and clinical characteristics in patients with NSCLC.

\begin{tabular}{|c|c|c|c|c|}
\hline Characteristics & CD147 (pg/mL) & $P$ & 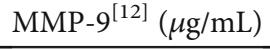 & $P$ \\
\hline Gender & & 0.959 & & 0.121 \\
\hline Male & $566.30(55.88-643.03)$ & & $2.80(1.08-6.96)$ & \\
\hline Female & $568.33(461.50-643.03)$ & & $2.29(0.80-3.59)$ & \\
\hline Age & & 0.676 & & 0.536 \\
\hline$\leq 60$ & $572.62(55.88-910.80)$ & & $2.61(0.80-5.49)$ & \\
\hline$>60$ & $551.34(281.00-822.25)$ & & $2.79(1.08-6.96)$ & \\
\hline Smoking status & & 0.637 & & 0.965 \\
\hline Never & $568.34(176.00-643.03)$ & & $2.87(1.34-4.84)$ & \\
\hline Smoker & $565.03(55.88-910.80)$ & & $2.74(0.80-6.96)$ & \\
\hline Baseline ECOG PS & & 0.543 & & 0.301 \\
\hline $0-1$ & $568.58(55.88-910.80)$ & & $2.74(0.80-6.96)$ & \\
\hline 2 & $554.35(510.80-781.11)$ & & $3.66(1.49-6.00)$ & \\
\hline Histological subtype & & 0.481 & & 0.499 \\
\hline Adenocarcinomas & $561.25(55.88-910.80)$ & & $2.81(1.08-6.00)$ & \\
\hline Squamous cell carcinomas & $571.36(281.00-822.25)$ & & $2.63(0.80-6.96)$ & \\
\hline T status & & 0.217 & & 0.928 \\
\hline $\mathrm{T} 1$ & $607.60(196.05-613.79)$ & & $2.17(1.82-4.02)$ & \\
\hline $\mathrm{T} 2$ & $554.35(434.00-692.02)$ & & $2.72(0.80-3.79)$ & \\
\hline $\mathrm{T} 3$ & $498.33(55.88-650.73)$ & & $2.51(1.49-6.96)$ & \\
\hline $\mathrm{T} 4$ & $597.16(176.00-910.80)$ & & $2.82(1.08-6.34)$ & \\
\hline $\mathrm{N}$ status & & 0.423 & & 0.800 \\
\hline No & $591.47(340.00-805.80)$ & & $2.55(1.82-4.20)$ & \\
\hline N1 & $314.89(55.88-573.90)$ & & $2.82(2.49-3.16)$ & \\
\hline $\mathrm{N} 2$ & $545.35(176.00-678.60)$ & & $2.83(1.49-4.84)$ & \\
\hline N3 & $562.82(281.00-910.80)$ & & $2.71(0.80-6.96)$ & \\
\hline M status & & 0.287 & & 0.497 \\
\hline M0 & $551.34(55.88-650.73)$ & & $2.72(0.80-6.96)$ & \\
\hline M1 & $569.84(176.00-910.80)$ & & $2.78(1.08-6.34)$ & \\
\hline TNM stage & & 0.287 & & 0.497 \\
\hline III & $551.34(55.88-650.73)$ & & $2.72(0.80-6.96)$ & \\
\hline IV & $569.84(176.00-910.80)$ & & $2.78(1.08-6.34)$ & \\
\hline
\end{tabular}

NCSCL: non-small-cell lung cancer; ECOG: Eastern Cooperative Oncology Group; PS: performance status.

MMP-9 levels before chemotherapy were not significantly associated with the clinical characteristics of the patients with NSCLC [12].

3.3. Association between the Changes in Serum Concentrations of CD147, MMP-9, and Tumor Responses in NSCLC. Serum concentrations of CD147 increased gradually after the first and second chemotherapy cycles and decreased after the fourth cycle (before chemotherapy $v s$. after the first chemotherapy cycle $(P=0.389)$, after the second chemotherapy cycle $(P=0.017)$, and after the fourth chemotherapy cycle $(P=0.444))$ (Figure 2(a)).

According to the chemotherapy response, we evaluated the difference in alterations in serum concentrations of CD147 in patients with different responses after the first, second, and fourth chemotherapy cycles. We assessed the changes in serum concentrations of CD147 in cases with $\mathrm{PD}$ and those with CR/PR/SD.
Compared with before $\mathrm{PD}$, serum concentrations of CD147 increased markedly in patients with PD. Compared with the previous time point of blood collection of cases with $\mathrm{CR} / \mathrm{PR} / \mathrm{SD}$, serum concentrations of CD147 increased slightly or declined in CR/PR/SD patients. The disparity of alterations in serum concentrations of CD147 in patients with different chemotherapy outcomes was significant after the first $(P=0.047)$ (Figure $2(\mathrm{~b}))$ and second chemotherapy cycles $(P=0.036)$ (Figure $2(c))$. However, there were no significant alterations in the serum concentrations of CD147 between cases who achieved PD and those with $\mathrm{CR} / \mathrm{PR} / \mathrm{SD}$ after the fourth chemotherapy cycle $(P=0.815)$ (Figure 2(d)).

The changes in serum concentrations of MMP-9 and tumor responses in NSCLC cases were performed with the same methods as our previous publication [12]. In PD patients, the serum level of MMP-9 increased before PD, and in CR/PR/SD patients, the serum level of MMP-9 was 


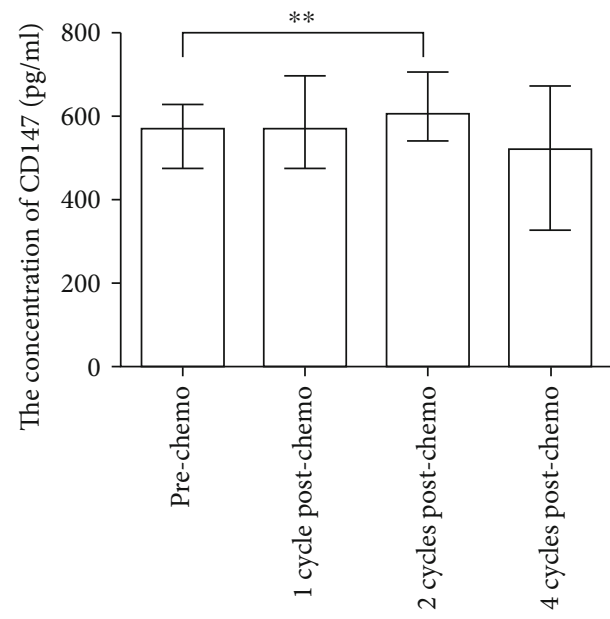

(a)

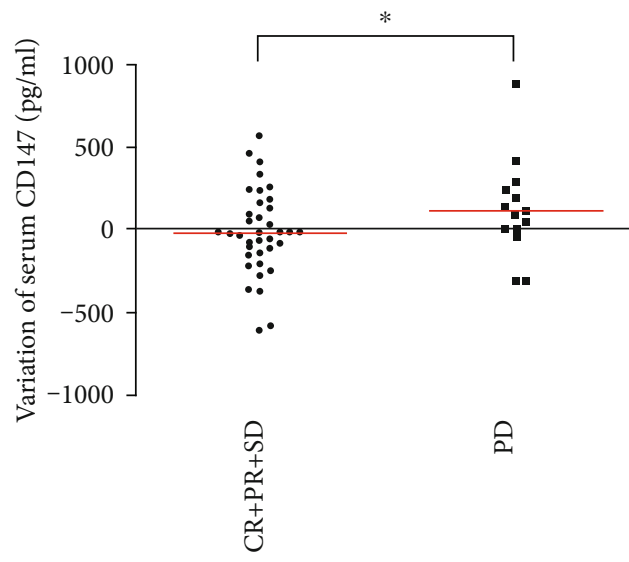

(c)

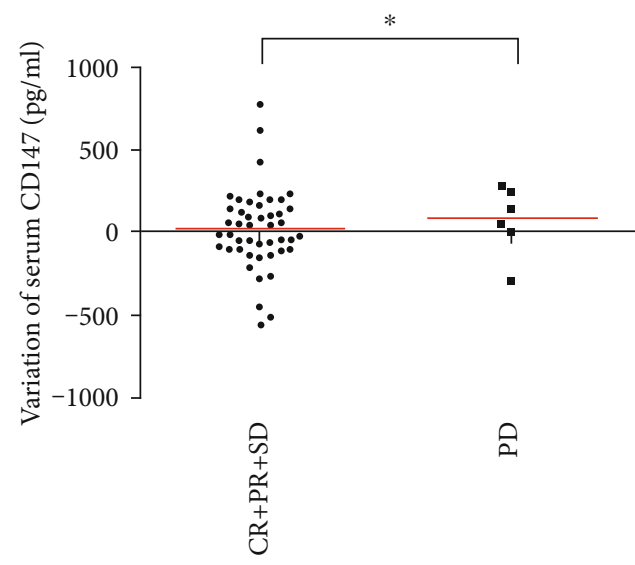

(b)

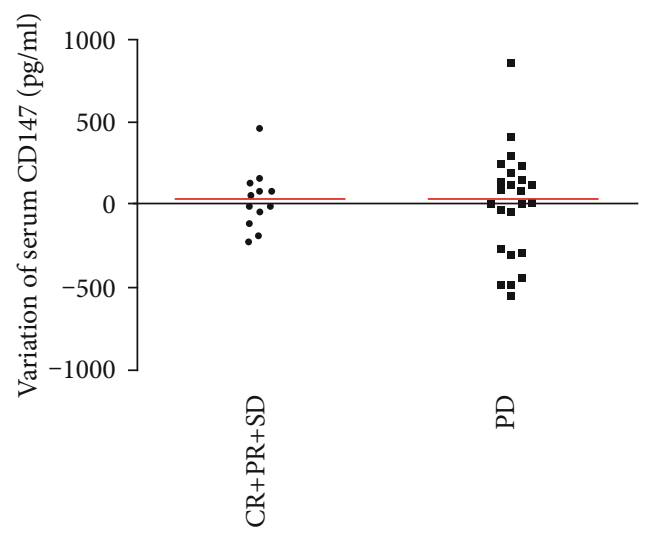

(d)

Figure 2: (a) Compared with baseline, serum concentrations of CD147 increased gradually after the first and second chemotherapy cycles and then decreased after the fourth cycle in patients with NSCLC. (b) Association between the changes in serum concentrations of CD147 from baseline to the first chemotherapy cycle and tumor response after the first chemotherapy cycle. (c) Association between the variation in serum concentrations of CD147 from the first to second chemotherapy cycle and tumor response after the second chemotherapy cycle. (d) Association between the alterations in serum concentrations of CD147 from the second to fourth chemotherapy cycle and tumor response evaluated after the fourth chemotherapy cycle. Each dot represents a patient, and red lines represent median values. CR: complete response; PR: partial response; SD: stable disease; PD: progressive disease. ${ }^{* *} P<0.01$ and ${ }^{*} P<0.05$.

decreased or stable. The difference in variation in serum MMP-9 levels in patients with different chemotherapy curative effects was statistically significant after one cycle, two cycles, and four cycles (after one cycle: $P<0.001$, after two cycles: $P<0.001$, and after four cycles: $P=0.01)[12]$.

3.4. The Combination of scCD147 and scMMP-9 as a Predictive Model for Chemotherapy Response. Based on the changes in scCD147 and scMMP-9 serum levels, upregulation and downregulation were considered positive and negative, respectively. The changes were compared with the clinical outcomes as shown in Table 2. Then, the predictive power of scCD147 and scMMP-9 was evaluated. The sensitivity of scCD147 and scMMP-9 alone was markedly higher than the specificity after one cycle, two cycles, and four cycles of chemotherapy (Figures 3(a) and 3(b)), indicating that the upregulation of scCD147 and scMMP-9 alone was beneficial for predicting PD but not CR/PR/SD. Furthermore, when
scCD147 and MMP-9 were combined into a new risk model, it had a markedly better prediction of efficacy than each protein alone, as shown in Figure 3(c). The specificity was significantly higher after one cycle, two cycles, and four cycles of chemotherapy, indicating that the combination of scCD147 and scMMP-9 could be beneficial for predicting CR/PR/SD.

3.5. Univariable and Multivariable Analyses for Tumor Response in NSCLC. Univariable analysis showed that variations in scMMP-9 $(P=0.021)$ after the first cycle, ECOG PS score $(P=0.021)$, regional lymph node metastasis $(P=0.035)$, variations in $\operatorname{scCD} 147(P=0.002)$, variations in scMMP-9 $(P=0.001)$ after the second cycle, variations in scCD147 $(P=0.045)$, and variations in scMMP-9 $(P=0.011)$ after the fourth cycle were significant factors for tumor response (Table S1). In addition, multivariable analysis demonstrated that variations in scCD147 (HR: 34.29, 95\% CI: 
TABLE 2: The comparison of serum CD147 and MMP-9 levels alone and combined with response to chemotherapy in patients with advanced NSCLC.

\begin{tabular}{|c|c|c|c|c|c|c|c|c|c|}
\hline \multirow[t]{2}{*}{ Parameter } & \multicolumn{2}{|c|}{$\begin{array}{l}\text { The first cycle } \\
\text { post-chemo }\end{array}$} & \multirow[t]{2}{*}{ Total } & \multicolumn{2}{|c|}{$\begin{array}{c}\text { The second cycle } \\
\text { post-chemo }\end{array}$} & \multirow[t]{2}{*}{ Total } & \multicolumn{2}{|c|}{$\begin{array}{c}\text { The fourth cycle } \\
\text { post-chemo }\end{array}$} & \multirow[t]{2}{*}{ Total } \\
\hline & $\mathrm{PD}$ & Non-PD & & $\mathrm{PD}$ & Non-PD & & $\mathrm{PD}$ & Non-PD & \\
\hline \multicolumn{10}{|l|}{ CD147 } \\
\hline Positive & 4 & 24 & 28 & 9 & 14 & 23 & 14 & 8 & 22 \\
\hline Negative & 2 & 23 & 25 & 4 & 23 & 27 & 11 & 6 & 17 \\
\hline Total & 6 & 47 & 53 & 13 & 37 & 50 & 25 & 14 & 39 \\
\hline \multicolumn{10}{|l|}{ MMP-9 } \\
\hline Positive & 6 & 15 & 21 & 10 & 27 & 37 & 21 & 9 & 30 \\
\hline Negative & 0 & 32 & 32 & 3 & 10 & 13 & 4 & 5 & 9 \\
\hline Total & 6 & 47 & 53 & 13 & 37 & 50 & 25 & 14 & 39 \\
\hline \multicolumn{10}{|c|}{ CD147+MMP-9 } \\
\hline Positive & 4 & 10 & 14 & 6 & 4 & 10 & 10 & 5 & 15 \\
\hline Negative & 2 & 37 & 39 & 7 & 33 & 40 & 15 & 9 & 24 \\
\hline Total & 6 & 47 & 53 & 13 & 37 & 50 & 25 & 14 & 39 \\
\hline
\end{tabular}

3.04-386.73; $P=0.004$ ), variations in scMMP-9 (HR: 32.83, 95\% CI: 3.23-333.90; $P=0.003)$ after the second cycle, and variations in scMMP-9 (HR: 12.00 , 95\% CI: 1.98-72.89; $P=0.007)$ after the fourth cycle may be independent factors to monitor chemotherapy outcome for patients with NSCLC (Table S2).

\section{Discussion}

Despite improvements in diagnostic and treatment methods, the prognosis of people with LC is still poor. The five-year survival of individuals with advanced LC is $\leq 15 \%$ [4]. Invasion and migration of tumor cells always result in tumor cells breaking through immunologic barriers such as blood and lymph $[13,14]$. Studies have indicated [15-18] that several factors are involved in the invasion/metastases of tumor cells. However, the key events involved in metastases are the degradation of the basement membrane of cells and ECM [19], and MMPs play crucial roles in these cellular mechanisms [20]. Recent studies have shown that CD147 promotes MMP formation by degrading the ECM of tumor cells to promote the invasion/metastases of tumor cells [21, 22], indicating that CD147 and MMPs form an axis that might be beneficial in the prognosis of chemotherapy. Studies have already shown several promising biomarkers for the prediction of chemotherapy for patients with NSCLC, such as excision repair cross-complementing group (ERCC), thymidylate synthase (TYMS), tubulin, and ribonucleotide reductase subunit M1 (RRM1) [23]. Previously in our study, we demonstrated that changes in serum concentrations of MMP-9 in patients with NSCLC during chemotherapy were intimately associated with chemotherapy outcome [12], which was in accordance with a previous study in colorectal cancer [24]. The sensitivity of scMMP-9 was markedly higher than the specificity, indicating that the upregulation of scMMP-9 was beneficial for predicting PD. However, the specificity of scMMP-9 was insufficient in monitoring $\mathrm{CR} / \mathrm{PR} / \mathrm{SD}$. The combination of two markers was supe- rior to either of them alone. Here, we studied the correlation between the changes in serum concentrations of CD147/MMP-9 and chemotherapy outcome in NSCLC cases.

In recent years, several studies have shown high concentrations of CD147 expressed in laryngeal cancer [25], oral cancer [26], lung cancer [27], breast cancer [28], myeloma [29], and colon cancer [30], which indicates that CD147 serum levels may be correlated with the development, invasion, and metastasis of tumor cells [31-33]. Ding and colleagues [34] conducted a meta-analysis to assess the association of CD147 expression in patients with hepatocellular carcinoma (HCC). They found CD147 expression to be potentially closely related to survival from HCC and associated clinicopathologic parameters.

Taken together, the results of this study showed that CD147 levels were significantly higher in patients with LC than those in HVs. Serum concentrations of CD147 in patients with LC were related to distant metastases and TNM stage. We demonstrated that the serum concentrations of CD147 have promising diagnostic and predictive efficacy prospects, an observation that is in accordance with previous studies.

We carried out sequential measurements of serum concentrations of CD147 in NSCLC cases in parallel with the evaluation of chemotherapy response, similar to our previous study that focused on MMP-9 [12]. In patients with NSCLC, serum concentrations of CD147 increased markedly in those who developed PD but increased slightly or decreased in those who achieved CR/PR/SD. The difference in the alterations in serum concentrations of CD147 in patients with different chemotherapy outcomes was significantly different between the first and second chemotherapy treatments. These findings suggested that sequential variations in the serum concentrations of CD147 could be beneficial for assessing chemotherapy outcome (i.e., CR/PR/SD or PD) in people with NSCLC. Serum concentrations of CD147 that decline or stabilize suggest CR/PR/SD, but those that increase denote PD. 


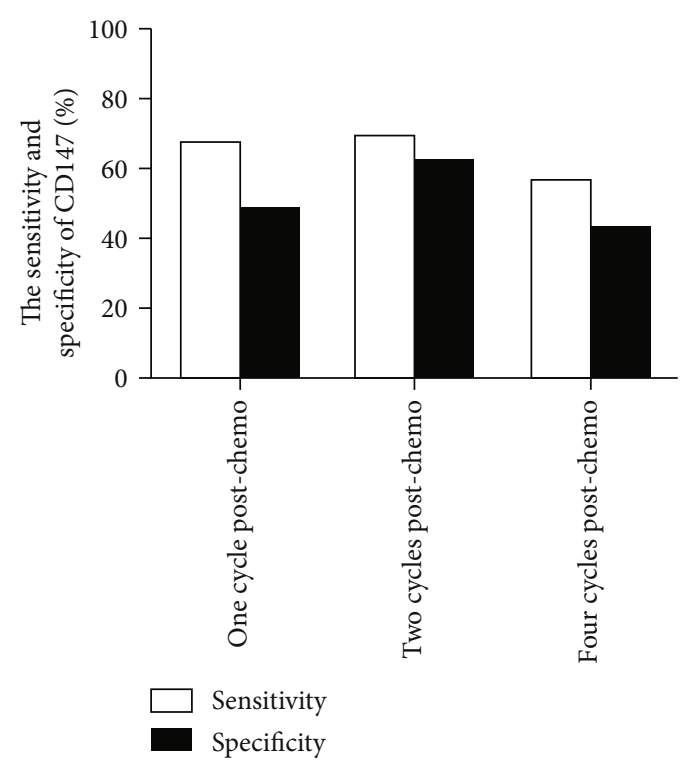

(a)

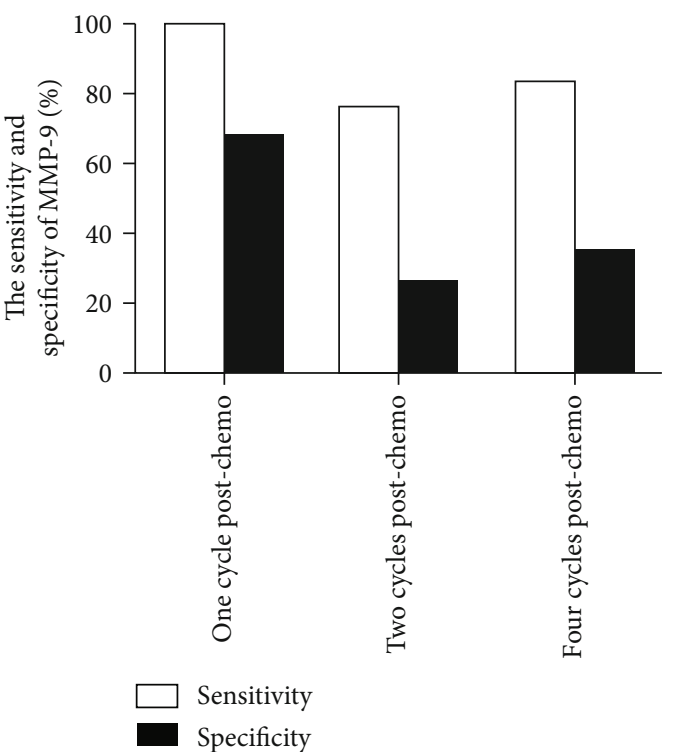

(b)

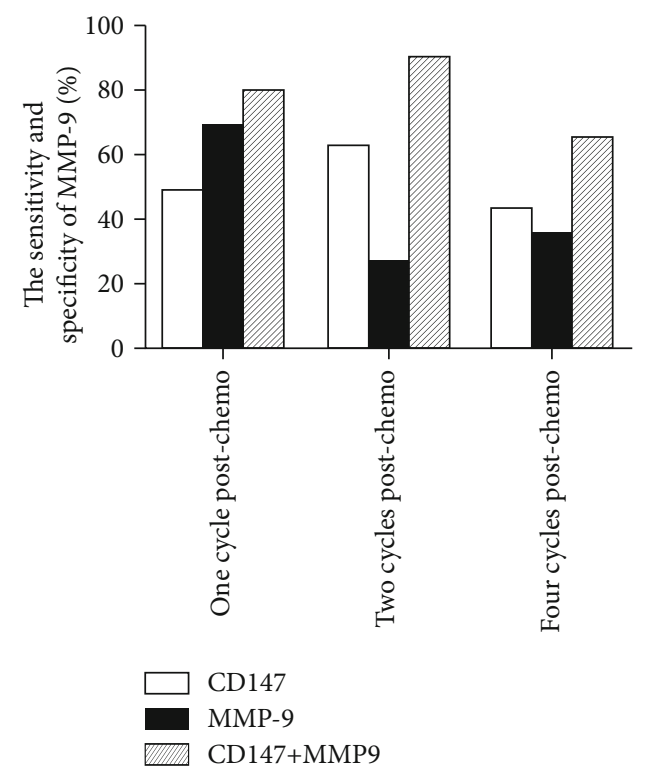

(c)

FIgURe 3: The combination of scCD147 and scMMP-9 as a predictive model for chemotherapy response. (a) The sensitivity and specificity of scCD147 were evaluated after one cycle, two cycles, and four cycles of chemotherapy. (b) The sensitivity and specificity of scMMP-9 were evaluated after one cycle, two cycles, and four cycles of chemotherapy. (c) The specificity of combined scCD147 and scMMP-9 was evaluated after one cycle, two cycles, and four cycles of chemotherapy.

Several studies have shown that CD147 and MMP-9 are related to the development, invasion, and metastasis of tumor cells. Infiltration of malignant tumor cells is aided by high expression of CD147, which can increase MMP-9 activity to cause degradation of the ECM and the basement membrane of cells $[35,36]$. Therefore, CD147 expression on tumor cell surfaces is, in general, considered to be increased, which leads to the stimulation of MMP-9 activity.

Similar to scMMP-9, the sensitivity of scCD147 alone was markedly higher than the specificity, and the upregulation of scCD147 alone was beneficial for predicting PD. When scCD147 and MMP-9 were combined into a new risk model, the specificity was significantly higher, indicating that the combination of scCD147 and scMMP-9 could be beneficial for predicting CR/PR/SD. These results suggested that sequential changes in the serum concentrations of CD147 and MMP-9 could be beneficial for assessing chemotherapy outcome (i.e., CR/PR/SD or PD) in patients with NSCLC.

Multivariable analysis demonstrated that variations in scCD147 and scMMP-9 after the second cycle and variations in scMMP-9 after the fourth cycle could be independent factors to monitor chemotherapy outcome for patients with NSCLC. scCD147 and scMMP-9 as a combination provide a new risk model that significantly improves the predictive 
power for chemotherapy response in patients with NSCLC. MMP-9 plus another protein as a combination is not a special case in our study; likewise, in colorectal cancer, scMMP-9 plus scRab1B also showed powerful predictive importance for chemotherapy outcomes [24].

Taken together, the combination of two biomarkers, CD147/MMP-9, seems to have promising benefits for the outcome of chemotherapy in patients with NSCLC. However, since the results of the aforementioned study were obtained in a retrospective single-institute study, they need to be validated in a future study involving a large number of patients in conjunction with determination of the optimal cut-off levels of biomarkers.

Serum concentrations of CD147 were (i) higher in LC cases than those in HVs, (ii) were associated with distant metastases and TNM stage in patients with LC, and (iii) may have useful diagnostic and predictive value for efficacy. Changes in serum concentrations of CD147 in patients with NSCLC during chemotherapy were intimately related to outcome. Serum concentrations of CD147 that fell or stabilized suggested CR/PR/SD, but increasing concentrations denoted PD. Variations in scCD147 and scMMP-9 may be independent factors for monitoring chemotherapy outcomes in patients with NSCLC. Furthermore, scCD147 and scMMP9 are potential predictive biomarkers for efficacy, and their combination significantly improves the predictive power for chemotherapy response in patients with NSCLC.

\section{Abbreviations}

CD147: Cluster of differentiation-147

MMP-9: Matrix metalloproteinase 9

LC: $\quad$ Lung cancer

ELISA: Enzyme-linked immunosorbent assay

ROC: Receiver operating characteristic

HVs: Healthy volunteers

NSCLC: Non-small-cell lung cancer

CR: $\quad$ Complete response

PR: Partial response

SD: $\quad$ Stable disease

PD: $\quad$ Progressive disease

SCLC: Small cell lung cancer

MMPs: Matrix metalloproteinases

ECM: Extracellular matrix

ECOG: Eastern Cooperative Oncology Group

PS: $\quad$ Performance status

SCC: $\quad$ Squamous cell carcinoma

TNM: Tumor, node, and metastases.

\section{Data Availability}

Answer: No. Comment: The datasets used and/or analyzed during the current study are available from the corresponding author on reasonable request.

\section{Additional Points}

Key Points. Changes in the serum concentrations of CD147 in patients with NSCLC during chemotherapy were intimately related to outcome. Serum concentrations of CD147 that fell or stabilized suggested CR/PR/SD, but increasing concentrations denoted PD. scCD147 and scMMP-9 are potential predictive biomarkers for efficacy, and their combination significantly improves the predictive power for chemotherapy response in patients with NSCLC.

\section{Ethical Approval}

All procedures involving human participants were in accordance with the ethical standards of the institutional and/or national research committee and with the 1964 Helsinki declaration and its later amendments or comparable ethical standards.

\section{Consent}

Informed consent was obtained from all individual participants included in the study.

\section{Conflicts of Interest}

The authors declare no conflict of interest.

\section{Authors' Contributions}

Xiaojuan Qiao, Yongfeng Jia, and Shucai Zhang are senior authors.

\section{Acknowledgments}

This investigation was funded by the Natural Science Foundation of Inner Mongolia (2016MS (LH) 0816) and was funded by the Doctoral Scientific Fund Project of the Affiliated Hospital of Inner Mongolia Medical University (NYFY BS 2018).

\section{Supplementary Materials}

Table S1: univariable analyses for tumor response in NSCLC cases after the various cycles of chemotherapy. Table S2: multivariable analyses for tumor response in NSCLC cases after the various cycles of chemotherapy. (Supplementary Materials)

\section{References}

[1] B. Fang, R. J. Mehran, J. V. Heymach, and S. G. Swisher, "Predictive biomarkers in precision medicine and drug development against lung cancer," Chinese Journal of Cancer, vol. 34, no. 7, pp. 295-309, 2015.

[2] R. Yang, P. Li, G. Zhang, C. Lu, H. Wang, and G. Zhao, "Long non-coding RNA XLOC_008466 functions as an oncogene in human non-small cell lung cancer by targeting miR-874," Cellular Physiology and Biochemistry, vol. 42, no. 1, pp. 126-136, 2017.

[3] B. K. Edwards, A. M. Noone, A. B. Mariotto et al., "Annual Report to the Nation on the Status of Cancer, 1975-2010, featuring prevalence of comorbidity and impact on survival among persons with lung, colorectal, breast, or prostate cancer," Cancer, vol. 120, no. 9, pp. 1290-1314, 2014. 
[4] M. Zeltsman, J. Dozier, E. McGee, D. Ngai, and P. S. Adusumilli, "CAR T-cell therapy for lung cancer and malignant pleural mesothelioma," Translational Research, vol. 187, pp. 1-10, 2017.

[5] B. Vogelstein, N. Papadopoulos, V. E. Velculescu, S. Zhou, L. A. Diaz, and K. W. Kinzler, "Cancer genome landscapes," Science, vol. 339, no. 6127, pp. 1546-1558, 2013.

[6] C. Biswas, Y. Zhang, R. DeCastro et al., "The human tumor cell-derived collagenase stimulatory factor (renamed EMMPRIN) is a member of the immunoglobulin superfamily," Cancer Research, vol. 55, no. 2, pp. 434-439, 1995.

[7] S. M. Agrawal and V. W. Yong, "The many faces of EMMPRIN-Roles in neuroinflammation," Biochimica et Biophysica Acta (BBA) - Molecular Basis of Disease, vol. 1812, no. 2, pp. 213-219, 2011.

[8] S. Suzuki, K. Honda, H. Nanjo et al., “CD147 expression correlates with lymph node metastasis in T1-T2 squamous cell carcinoma of the tongue," Oncology Letters, vol. 14, no. 4, pp. 4670-4676, 2017.

[9] D. Urbaniak-Kujda, K. Kapelko-Slowik, I. Prajs et al., "Increased expression of metalloproteinase-2 and -9 (MMP2, MMP-9), tissue inhibitor of metalloproteinase-1 and -2 (TIMP-1, TIMP-2), and EMMPRIN (CD147) in multiple myeloma," Hematology, vol. 21, no. 1, pp. 26-33, 2016.

[10] L. Huang, A. M. Xu, and Q. Peng, "CD147 and MMP-9 expressions in type II/III adenocarcinoma of esophagogastric junction and their clinicopathological significances," International Journal of Clinical and Experimental Pathology, vol. 8, no. 2, pp. 1929-1937, 2015.

[11] H. Yin, Y. Shao, and X. Chen, "The effects of CD147 on the cell proliferation, apoptosis, invasion, and angiogenesis in glioma," Neurological Sciences, vol. 38, no. 1, pp. 129-136, 2017.

[12] X. Qiao, X. Zhai, J. Wang et al., "Sequential measurements of serum matrix metalloproteinase 9 to monitor chemotherapy responses in patients with advanced non-small-cell lung cancer," OncoTargets and Therapy, vol. 9, pp. 3299-3305, 2016.

[13] L. Du, X. Chen, Y. Cao et al., "Overexpression of PIK3CA in murine head and neck epithelium drives tumor invasion and metastasis through PDK1 and enhanced TGF $\beta$ signaling," Oncogene, vol. 35, no. 35, pp. 4641-4652, 2016.

[14] M. J. Sorich, A. Rowland, C. S. Karapetis, and A. M. Hopkins, "Evaluation of the lung immune prognostic index for prediction of survival and response in patients treated with atezolizumab for NSCLC: pooled analysis of clinical trials," Journal of Thoracic Oncology, vol. 14, no. 8, pp. 1440-1446, 2019.

[15] G. Song, H. Hsiao, J. L. Wang et al., "Differential impact of tumor-infiltrating immune cells on basal and luminal cells: implications for tumor invasion and metastasis," Anticancer Research, vol. 34, no. 11, pp. 6363-6380, 2014.

[16] R. Saito, R. Shirakawa, H. Nishiyama et al., "Downregulation of Ral GTPase-activating protein promotes tumor invasion and metastasis of bladder cancer," Oncogene, vol. 32, no. 7, pp. 894-902, 2013.

[17] Y. Liu, Y. D. Zhou, Y. L. Xiao et al., "Cyr61/CCN1 overexpression induces epithelial-mesenchymal transition leading to laryngeal tumor invasion and metastasis and poor prognosis," Asian Pacific Journal of Cancer Prevention, vol. 16, no. 7, pp. 2659-2664, 2015.

[18] M. Ito, K. Teshima, S. Ikeda et al., "MicroRNA-150 inhibits tumor invasion and metastasis by targeting the chemokine receptor CCR6, in advanced cutaneous T-cell lymphoma," Blood, vol. 123, no. 10, pp. 1499-1511, 2014.

[19] M. Yilmaz, G. Christofori, and F. Lehembre, "Distinct mechanisms of tumor invasion and metastasis," Trends in Molecular Medicine, vol. 13, no. 12, pp. 535-541, 2007.

[20] J. Vandooren, P. E. Van den Steen, and G. Opdenakker, "Biochemistry and molecular biology of gelatinase B or matrix metalloproteinase-9 (MMP-9): the next decade," Critical Reviews in Biochemistry and Molecular Biology, vol. 48, no. 3, pp. 222-272, 2013.

[21] X. Gou, H. Chen, F. Jin et al., "Expressions of CD147, MMP-2 and MMP-9 in laryngeal carcinoma and its correlation with poor prognosis," Pathology Oncology Research, vol. 20, no. 2, pp. 475-481, 2014.

[22] J. Remon, M. J. Ahn, N. Girard et al., "Advanced-stage nonsmall cell lung cancer: advances in thoracic oncology 2018," Journal of Thoracic Oncology, vol. 14, no. 7, pp. 1134-1155, 2019.

[23] D. Liu, N. Nakashima, J. Nakano et al., "Customized adjuvant chemotherapy based on biomarker examination may improve survival of patients completely resected for non-small-cell lung cancer," Anticancer Research, vol. 37, no. 5, pp. 2501-2507, 2017.

[24] X.-Z. Yang, S. Z. Cui, L. S. Zeng et al., "Overexpression of Rab1B and MMP9 predicts poor survival and good response to chemotherapy in patients with colorectal cancer," Aging, vol. 9, no. 3, pp. 914-931, 2017.

[25] S. Suzuki, M. Sato, H. Senoo, and K. Ishikawa, "Direct cell-cell interaction enhances pro-MMP-2 production and activation in co-culture of laryngeal cancer cells and fibroblasts: involvement of EMMPRIN and MT1-MMP," Experimental Cell Research, vol. 293, no. 2, pp. 259-266, 2004.

[26] V. Richard, P. Sebastian, M. G. Nair et al., "Multiple drug resistant, tumorigenic stem-like cells in oral cancer," Cancer Letters, vol. 338, no. 2, pp. 300-316, 2013.

[27] H. Z. Zeng, Y. Q. Qu, A. B. Liang et al., "Expression of CD147 in advanced non-small cell lung cancer correlated with cisplatin-based chemotherapy resistance," Neoplasma, vol. 58, no. 5, pp. 449-454, 2011.

[28] L.-M. Kong, C. G. Liao, Y. Zhang et al., "A regulatory loop involving miR-22, Sp1, and c-Myc modulates CD147 expression in breast cancer invasion and metastasis," Cancer Research, vol. 74, no. 14, pp. 3764-3778, 2014.

[29] S. Panchabhai, K. Kelemen, G. Ahmann, S. Sebastian, J. Mantei, and R. Fonseca, "Tumor-associated macrophages and extracellular matrix metalloproteinase inducer in prognosis of multiple myeloma," Leukemia, vol. 30, no. 4, pp. 951954, 2016.

[30] L. Gao, L. Shen, M. Yu et al., "Colon cancer cells treated with 5-fluorouracil exhibit changes in polylactosamine-type N-glycans," Molecular Medicine Reports, vol. 9, no. 5, pp. 1697-1702, 2014.

[31] Z. Zhang, Y. Zhang, X. X. Sun, X. Ma, and Z. N. Chen, "microRNA-146a inhibits cancer metastasis by downregulating VEGF through dual pathways in hepatocellular carcinoma," Molecular Cancer, vol. 14, no. 1, p. 5, 2015.

[32] Z. Yan, K. Qu, J. Zhang et al., "CD147 promotes liver fibrosis progression via VEGF-A/VEGFR2 signallingmediated cross-talk between hepatocytes and sinusoidal endothelial cells," Clinical Science, vol. 129, no. 8, pp. 699710,2015 
[33] F. Bougatef, S. Menashi, F. Khayati et al., "EMMPRIN promotes melanoma cells malignant properties through a HIF-2alpha mediated up-regulation of VEGF-receptor-2," PLoS One, vol. 5, no. 8, article e12265, 2010.

[34] P. Ding, X. Zhang, S. Jin et al., "CD147 functions as the signaling receptor for extracellular divalent copper in hepatocellular carcinoma cells," Oncotarget, vol. 8, no. 31, pp. 51151-51163, 2017.

[35] W. D. Zhong, Z. D. Han, H. C. He et al., "CD147, MMP-1, MMP-2 and MMP-9 protein expression as significant prognostic factors in human prostate cancer," Oncology, vol. 75, no. 3-4, pp. 230-236, 2008.

[36] S. Piao, S. Zhao, F. Guo et al., "Increased expression of CD147 and MMP-9 is correlated with poor prognosis of salivary duct carcinoma," Journal of Cancer Research and Clinical Oncology, vol. 138, no. 4, pp. 627-635, 2012. 